\title{
ACEPTACIÓN DE MEDICAMENTOS GENÉRICOS POR USUARIOS DE SECTORES C, D Y E EN TRES ZONAS DE LIMA
}

\author{
Acceptance of generic drugs by users from sector C, D and E sectors in three zones of Lima \\ Loly F Canales $\mathrm{M}^{1}$, Jesús V Rumiche $\mathrm{B}^{1}$ \\ ${ }^{1}$ Facultad de Farmacia y Bioquímica, UNMSM
}

\section{RESUMEN}

Esta investigación tuvo como objetivo determinar el grado de aceptación de medicamentos genéricos (MG) por parte de usuarios pertenecientes a los sectores C, D y E, de tres zonas de Lima Metropolitana, así como el grado de conocimiento, confianza y las posibles barreras en su consumo; para ello se aplicó una encuesta en las zonas donde más del $85 \%$ de la población pertenece a estos sectores socio-económicos. El instrumento fue un cuestionario de 11 preguntas abiertas y cerradas. Se identificaron cuatro dimensiones que influyen en la aceptación de los medicamentos genéricos: información, seguridad/eficacia, consumo y acceso. El análisis de los resultados obtenidos por la escala de Stanones, estableció una media promedio de 3,2 entre las 4 dimensiones, lo que se interpreta como una dimensión REGULAR. Se encontró un alto consumo de medicamentos genéricos, con una media de 4,9; accesibilidad ligada más a la capacidad económica que a la confianza hacia este tipo de medicamento, pues existen dudas sobre su seguridad y eficacia.

Palabras claves: Medicamento genérico, aceptabilidad.

\section{SUMMARY}

The aim of this research was to determine the acceptability of generic drugs (GD) among users that belong to the sectors C, D and E in Metropolitan Lima, as well as the grade of knowledge, confidence and possible barriers in its consumption. A survey was conducted in zones where over than the $85 \%$ of the population belongs to this sectors .The tool consisted in a questionnaire of 11 open and closed questions. It was identified four dimensions that influenced on the acceptance of the generic drugs: information, security/efficacy, consumption and access. The analysis of the results obtained by the scale of Stanones set the media average of 3,2 among the four dimensions whichs means a REGULAR dimension. It was found a high consumption of GD with a media of 4,9; this accessibility is linked to the low cost instead than confidence in the GD, since there are doubts about its efficiency.

Keywords: generic drug, acceptability.

\section{INTRODUCCIÓN}

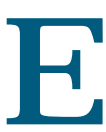
l sistema de salud esta compuesto por tres pilares fundamentales: los recursos humanos, es decir los profesionales de salud y administración en salud, la infraestructura y los medicamentos. Estos últimos deben estar disponibles y ser accesibles para toda la población; para promover esto se debería lograr que el precio de los medicamentos estéal alcance de los sectores con menores recursos, que son los más afectados. Los medicamentos genéricos juegan un papel fundamental en la asequibilidad, es por ello que la imagen y aceptación que tengan en la población es relevante en salud publica ${ }^{(1)}$.

Un elemento central para la incorporación de las políticas de MG es garantizar su aceptación por parte de la sociedad, es decir, la consolidación de una oferta relativamente adecuada a las necesidades de salud de una población no tendrá impacto positivo si las personas no demandan los productos ${ }^{(3)}$. Por ello, el objetivo es determinar el grado de aceptación de los MG por usuarios pertenecientes a los sectores C, D y E, de tres zonas de Lima Metropolitana, así como el grado de conocimiento, confianza y las posibles barreras en su consumo.

\section{MATERIALES Y MÉTODOS}

Estudio descriptivo de corte transversal realizado en los sectores C, D y E en tres zonas de Lima Metropolitana.

\section{Universo}

Se seleccionaron 3 de las 10 zonas en las que se fraccionó a Lima Metropolitana en el estudio del 2010 de la Asociación Peruana de Empresas de Investigación de Mercado (APEIM) ${ }^{(4)}$. Estas tres zonas representaron el $32,3 \%$ del nivel socio económico C, el 42,4\% del nivel socio económico D y el 53,3\% del nivel socio económico $\mathrm{E}$. 
Se determinó el número de individuos mayores de 18 años en los 10 distritos contenidos en las tres zonas, utilizando los Censos Nacionales 2007: XI de Población y VI de Vivienda proveniente del Instituto Nacional de Estadística e Informática (INEI) ${ }^{(5)}$.

\section{Muestra}

Seaplicó un muestreo probabilístico proporcional estratificado.

Criterios de inclusión: Usuarios de servicios de salud estatales y establecimientos privados, mayores de 18 años, habitantes de la zona, excluyendo a aquellos que no acepten participar voluntariamente.

La muestra abarca 426 individuos distribuidos proporcionalmente al tamaño de la población de cada distrito.

\section{Ubicación de los centros de salud y conglomerados de farmacias}

Se ubicaron los hospitales y conglomerados de farmacias en los diez distritos que conformaban las tres zonas, asegurando su ubicación en manzanas correspondientes a los sectores que abarca el estudio, en los planos estratificados del INEI (6).

\section{Aplicación de la encuesta}

Se aplicó la encuesta asistida a los usuarios de servicios de salud estatales y establecimientos privados durante los meses de diciembre del 2010, enero y febrero del 2011, en los distritos que conforman las tres zonas en estudio, al momento que salían de dichos establecimientos.

En hospitales, la encuesta se aplicó a la salida, de los servicios de atención ambulatoria, luego de abandonar el establecimiento. Los participantes más colaboradores se captaron a la salida de su atención médica y de los servicios de farmacia. En el caso de farmacias y boticas, el usuario encuestado es abordado luego de salir del establecimiento.

Se solicita, amablemente al encuestado, su opinión acerca de los $\mathrm{MG}$, iniciándose las preguntas luego de su aceptación. El procedimiento se realizó todos los días de la semana, obteniéndose un promedio de 10 a 15 encuestas por día. La encuesta duró, en promedio, 8 minutos en ser culminada.

\section{RESULTADOS Y DISCUSIÓN}

Se encontró que $98,42 \%$ de la población en las tres zonas donde se realizó la medición, consumía los MG (figura 1); sin embargo, más de la mitad de los usuarios

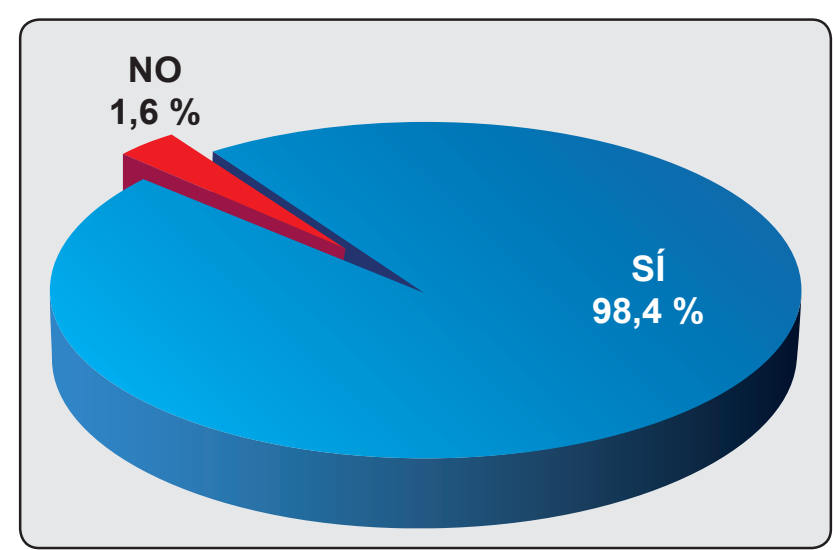

Figura 1. Consumo de medicamentos genéricos.

reportaron tener dudas sobre su seguridad y eficacia; esta dimensión obtuvo una media de 3,1, lo que revela temor o preocupación regular sobre la seguridad y/o eficacia de estos medicamentos. La razón para optar por los MG fue el bajo precio o su dispensación gratuita en centros de salud pertenecientes a ESSALUD y al MINSA. A partir de los resultados de las encuestas se puede afirmar que en los diez distritos donde se realizó el estudio, que representan el 32,3\%; 42,4\%; 53,3\% de la población de los sectores $\mathrm{C}$, $\mathrm{D}$ y E, respectivamente de Lima Metropolitana (Tabla 1), casi la totalidad consume MG, sin embargo, esta conducta obedece a factores económicos y no a una confianza en el medicamento genérico.

Los resultados de la pregunta "si aceptarían el cambio de un medicamento de marca por un genérico" (Figura 2) muestran la influencia que la prescripción médica tiene en la demanda de los medicamentos. El 41,1\% de encuestados no acepta el cambio de un medicamento de marca, prescrito por el médico, a un genérico, frente a un 33,2\% que definitivamente si lo aceptaría; esto obedecería al poco o nulo conocimiento que tiene el paciente cuando de medicamentos se trata. A esto se le suma el gran número de medicamentos que se consumen prescindiendo de la prescripción médica y siguiendo la recomendación del dependiente de la farmacia o botica, que casi en ningún caso favorecerá

Tabla 1. Distribución porcentual de NSE en las 3 zonas.

\begin{tabular}{lccc}
\hline \multicolumn{1}{c}{ Nivel Socioeconómico } & C (\%) & D (\%) & E (\%) \\
\hline $\begin{array}{l}\text { ZONA 1 (Puente Piedra, } \\
\text { Comas, Carabayllo) }\end{array}$ & 10,7 & 15,6 & 16,7 \\
$\begin{array}{l}\text { ZONA 3 (San Juan de } \\
\text { Lurigancho) }\end{array}$ & 11,0 & 12,2 & 17,0 \\
$\begin{array}{l}\text { ZONA 5 (Ate Vitarte, } \\
\text { Chaclacayo, Lurigancho, Santa }\end{array}$ & 10,6 & 14,6 & 19,6 \\
$\begin{array}{l}\text { Anita, San Luis, El Agustino) } \\
\text { TOTAL }\end{array}$ & 32,3 & 42,4 & 53,3 \\
\hline
\end{tabular}

Fuente: APEIM Niveles socioeconómicos de Lima Metropolitana, Enero 2010 
al consumo del MG, esto se hace visible en los resultados de la pregunta que evalúa el conocimiento sobre el MG, donde el 34,6\% de la muestra no sabe lo que es un MG (figura 3).

Un 36,8\% (figura 4) manifestó que le era difícil encontrar MG en las farmacias o boticas y un $24,1 \%$ señala que le es difícil a veces, esta dimensión denominada acceso, obtuvo una media de 3,0, lo que revela una dificultad regular, esto puede deberse a la integración vertical que tienen los puntos de venta con los laboratorios, donde inclusive la misma farmacia o botica dispensa medicamentos con

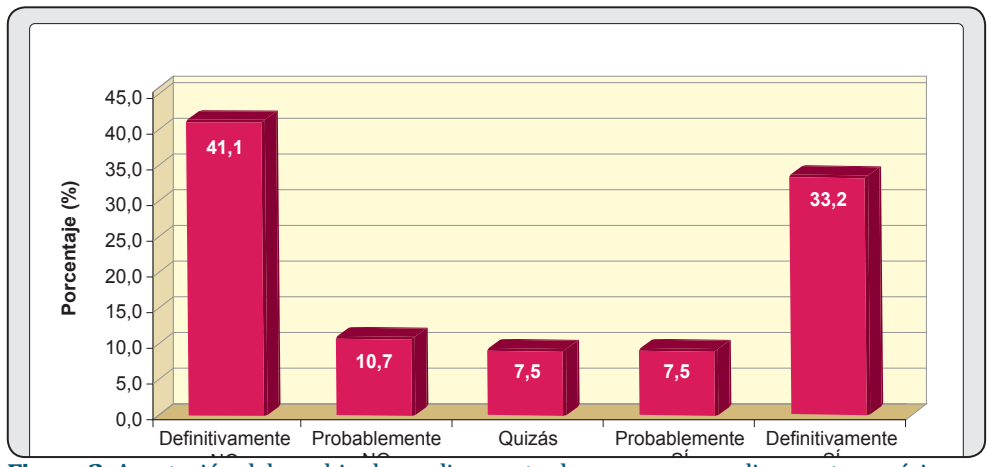

Figura 2. Aceptación del cambio de medicamento de marca por medicamento genérico en farmacias. marca propia hacia la cual trata de inclinar la demanda. Esta claro que el margen de ganancia que deja un MG es mínimo comparado con su similar de marca, por ello no es de extrañar que el dependiente de la farmacia trate que la mayor parte de sus ventas sean medicamentos de marca para poder cumplir su cuota de venta mensual; solo se dispensa el genérico si el cliente lo exige. Se hace más difícil si la compra no es en el día, las farmacias que hacen doble turno en la madrugada solo ofrecen medicamentos de marca.

Una medida para incentivar la demanda, en otros países, es el sistema de precio de referencia. Este sistema funciona fijando el precio de un producto en el mercado como de referencia, cuando el paciente acude a adquirir cierto medicamento a la farmacia se le dispensa el que tenga el precio de referencia, costo que es cubierto por el seguro de salud, si el paciente desea un medicamento de marca que sea más caro, la diferencia debe ser costeada por el propio paciente. Esto, obviamente, sólo funciona en sistemas donde la mayoría de la población cuenta con seguro, como ocurre en casi todos los países europeos. En España, el precio que es tomado como referencia es el precio del medicamento genérico ${ }^{(6)}$.

En los sectores donde se hizo la medición, el mayor riesgo encontrado en el consumo del medicamento genérico fue que el $47 \%$ (Figura 5) cree que no es un medicamento seguro y confiable; otro $15 \%$ (Figura 5) presenta dudas; el $42 \%$ (Figura 6 ) teme que no pueda proporcionarle los beneficios esperados, aunque esto no significa que no lo consuman, significa que no están satisfechos con el producto que consumen; un 4,0\% (Figura 7) manifestó que los consume porque considera que son iguales, la mayoría lo hace porque son más económicos o porque los reciben gratuitamente en los sistemas estatales de salud, esto quiere decir que si la situación económica de estas personas cambiara positivamente optarían por consumir un medicamento de marca, por

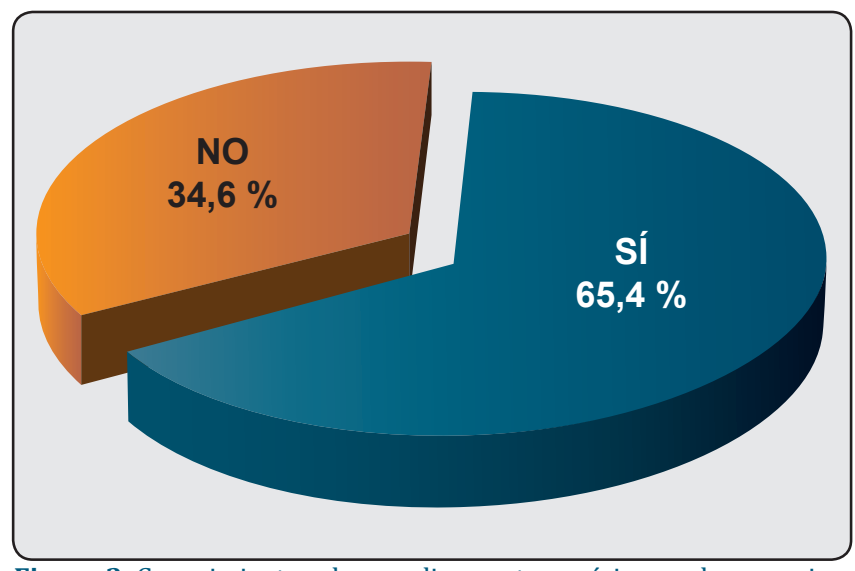

Figura 3. Conocimiento sobre medicamento genérico por los usuarios.

considerarlo un producto de mayor calidad, tal como ocurre en los estratos de mayor nivel socioeconómico, donde el factor económico no es un freno.

Por otro lado, encontramos una relación entre las preguntas 5, 6 y 7, donde el 33,2\% sí aceptaría el cambio por un genérico en la farmacia; este porcentaje es similar a los que no desconfían de su seguridad $(30,4 \%)$ y eficacia (37,5\%), concluyéndose que alrededor del $30 \%$ opina que son iguales, aunque no

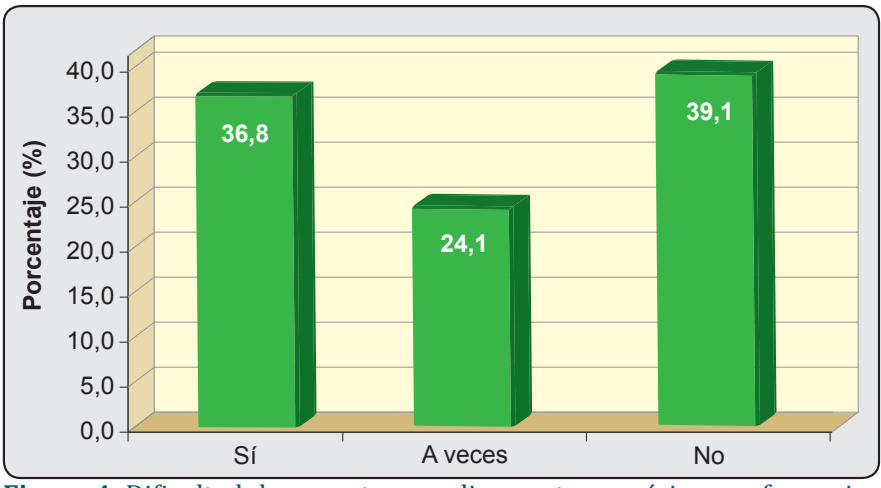

Figura 4. Dificultad de encontrar medicamentos genéricos en farmacias y boticas. 


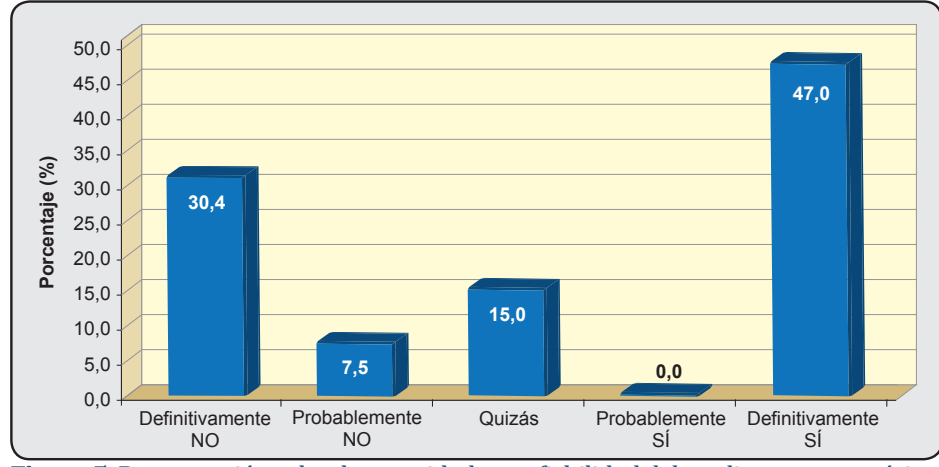

Figura 5. Preocupación sobre la seguridad y confiabilidad del medicamento genérico.

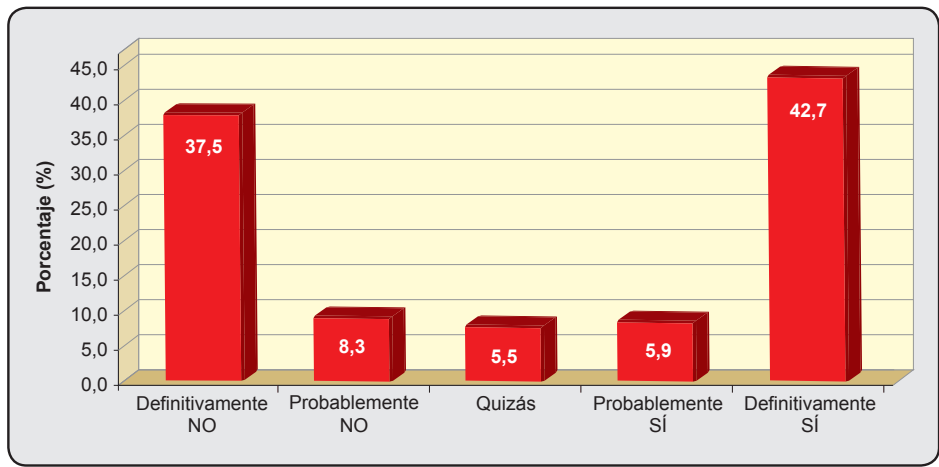

Figura 6. Temor de no obtener los beneficios que se esperan del medicamento por ser genérico.

identifica a esta cualidad como la determinante para consumirlos, lo hacen porque son más accesibles, según se observa en la figura 7. Muchos de los entrevistados además agregaron que, si su situación económica se lo permitiese en el momento de la compra, optarían por un similar de marca ya que hace efecto con mayor rapidez.

Para que el desarrollo del medicamento genérico sea sostenible, el mayor beneficio observado en este no debería ser el menor precio, de tomarlo de esa forma sólo prevalecería entre los medicamentos genéricos aquel que cueste menos, pasando por alto otros atributos que son de importancia para el consumidor final. Además, esto promovería un suministro del tipo monopólico, pues favorece al proveedor más grande capaz de ofrecer precios sin competencia. Un abastecimiento múltiple de medicamentos genéricos, permite escoger el producto con mejores características, además de asegurar su bajo precio a largo plazo al evitar la retirada de los competidores.

Aunque el Perú atraviesa por un buen momento económico, es un mercado relativamente pequeño, donde el consumo de grandes volúmenes que justifiquen la entrada de la versión genérica de un medicamento no siempre se da, el bajo volumen de la demanda potencial limita sus oportunidades comerciales; esto sucede porque al vencer la patente de una molécula no ingresa al mercado un medicamento genérico sino uno de marca, a diferencia de otros países con mercados mayores.

La demanda, en el caso especial de los medicamentos, no varía proporcionalmente con las variaciones de precio. En una situación de libre mercado y en presencia de una industria farmacéutica altamente concentrada, deja al consumidor en una situación de gran vulnerabilidad ${ }^{(2,7)}$. En consecuencia, ante la entrada de un producto más barato, que es el genérico, la demanda no se incrementaría, esta se mantendría o seguiría su crecimiento natural obedeciendo al incremento de la población y al envejecimiento de la misma; entonces, necesariamente para que un medicamento genérico tenga mayor participación de mercado tendría que quitarle participación a un medicamento similar.

Centrar excesivamente las ventajas del medicamento genérico en su bajo precio en un mercado relativamente pequeño como el peruano puede resultar contraproducente y podría ser la razón por la que a pesar de existir moléculas que no se encuentran bajo protección de patente en el territorio o cuya patente ya expiró, el genérico no ingresa inmediatamente. Tal es así, que en el Perú no se encuentran muchos genéricos en asociaciones como en otros países, es por ello que los beneficios sinérgicos de un polifármaco es asociado a los medicamentos de marca, en el Perú el consumidor no distingue entre

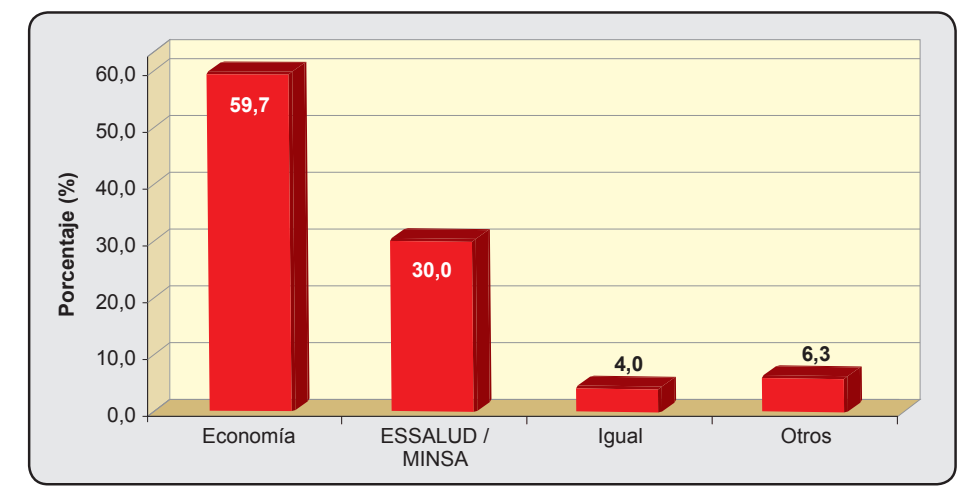

Figura 7. Razón del consumo de medicamentos genéricos 


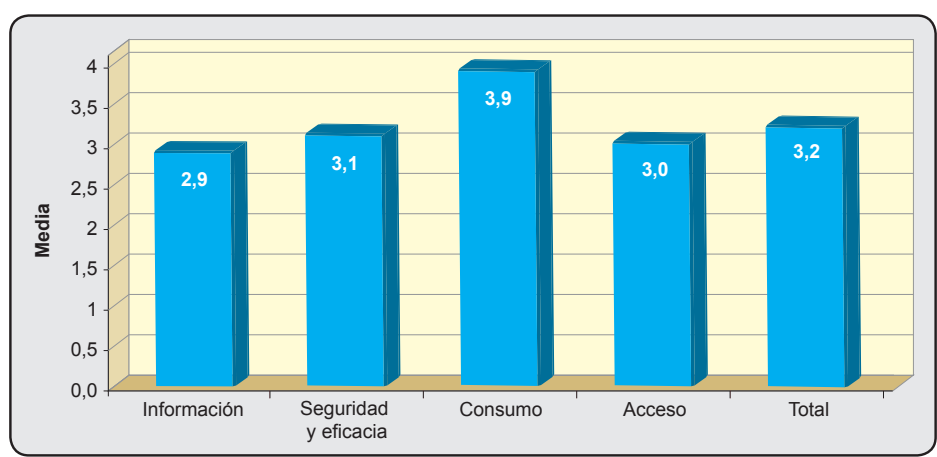

Figura 8. Media de las dimensiones de la aceptación de medicamentos genéricos.

moléculas, solo lo distingue por acción terapéutica y siempre van a ser más efectivos los fármacos asociados (polifármacos), este desconocimiento afecta la imagen del medicamento genérico indirectamente, haciéndolo ver menos efectivo que su similar de marca.

Este problema es causado por la asimetría de información que, como hemos visto, afecta al consumidor final a pesar de ser el actor más interesado en el producto, es el menos enterado de las características de este, limitando su poder de elección. En síntesis, quienes eligen el producto que se consume (los que los prescriben) no lo financian y quienes lo consumen (los pacientes) y financian (pacientes y aseguradores) no lo eligen. Los oferentes (laboratorios productores) suelen ser los únicos que poseen la información más completa en tiempo y forma, en tanto que el paciente, como consumidor, se ubica en el extremo opuesto ${ }^{(2)}$.

La difusión de la información es fundamental para el desarrollo sostenible del medicamento genérico en el Perú. La publicidad, que no es la única vía ni la más recomendable, aunque sí efectiva, debería cumplir una función educativa y estar a cargo del estado, pues ningún otro estaría interesado en impulsar el consumo del medicamento genérico. En nuestra medición el $71 \%$ refirió no haber recibido información por parte del estado sobre medicamentos y es preocupante ver que el 34,6\% no ha oído siquiera sobre los medicamentos genéricos. Aunque el estado queda en tercer lugar en las preferencias, observamos que este podría hacer una asociación publicitaria con los médicos, quienes quedaron en primer lugar, y los químicos farmacéuticos, ubicados en el segundo lugar, por medio de spots lanzados en televisión, que es el medio con mayor audiencia, y que supero largamente en preferencias a los otros medios de comunicación en nuestro sondeo, para educar permanentemente y con sostenibilidad al consumidor sobre los medicamentos genéricos. Esta etapa publicitaria deberá ser la parte culminante del plan de desarrollo del medicamento genérico en el Perú, solo después de haber asegurado su calidad a través de los organismos encargados (DIGEMID), de lo contrario podría generar inseguridad hacia el producto al correr el riesgo de comercializar unidades genéricas de dudosa calidad.

Finalmente, el análisis por dimensiones (Figura 8) arroja que en general existe una aceptación regular de los medicamentos genéricos en los sectores $\mathrm{C}, \mathrm{D}$ y $\mathrm{E}$ de las tres zonas abarcadas. La media más baja correspondió a si el estado brinda información sobre medicamentos genéricos, obteniéndose el valor de 2,1 que indica mala difusión de información. Asimismo, la media más alta correspondió al consumo de medicamentos genéricos, obteniéndose el valor de 4,9; este ítem pertenece a la dimensión de consumo que obtuvo una media de 3,9, lo que indica un buen consumo, sin embargo, las preguntas que miden la dimensión de seguridad y eficacia arrojan una media de 3,1, que indican temor y/o preocupación regular, con lo que se puede afirmar que el consumo esta ligado a un tema de asequibilidad.

\section{CONCLUSIONES}

1. El grado de aceptación de los medicamentos genéricos es regular, según escala Stanones $x_{4}=3,2$.

2. Se encuentra un déficit en la información sobre los medicamentos genéricos. El estado no cumple un buen papel en la difusión de la información.

3. Existe desconfianza al medicamento genérico en los sectores en estudio (47\%) aunque ello no influye negativamente en el consumo, ya que este último se encuentra más influenciado por otros factores como la asequibilidad.

4. La dificultad para el acceso al medicamento genérico es regular en los puntos de venta del sector privado de los sectores $\mathrm{C}$, D y E de las tres zonas estudiadas.

5. El $41,1 \%$ definitivamente no y el $10,7 \%$ probablemente no está dispuesto a optar por un medicamento genérico si el médico prescribe un medicamento de marca.

6. El médico es el profesional con mayor influencia en el paciente $(69 \%)$ seguido por el químico farmacéutico con $17,1 \%$.

7. El medio de comunicación preferido para informarse sobre el medicamento es el televisivo con $64,9 \%$ seguido muy de lejos por la radio con un $12,7 \%$. 
ENCUESTA ACEPTACIÓN DE MEDICAMENTOS GENÉRICOS \begin{tabular}{|l|l|l|l|l|l|l|l|l|l|}
\hline 1 & 2 & 3 & 4 & 5 & 6 & 7 & 8 & 9 & 10 \\
\hline
\end{tabular}

1 ¿En que distrito vive?

Puente Piedra

Comas

Carabayllo

San Juan de Lurigancho

Ate Vitarte

Chaclacayo

Lurigancho

Santa Anita

San Luis

El Agustino

2 ¿Ha oído hablar de los medicamentos genéricos? (Si la respuesta es no pasar a la pregunta 10)

Sí

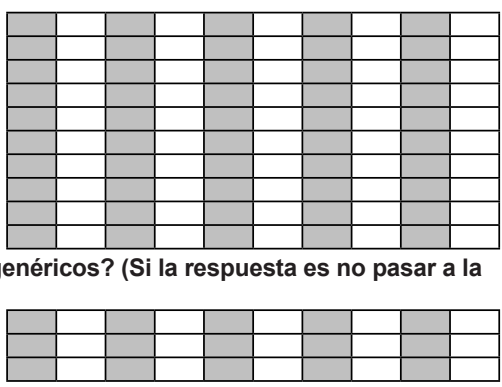

3 ¿Consume medicamentos genéricos? ¿Por qué?

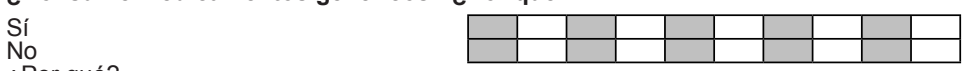

¿Por qué?

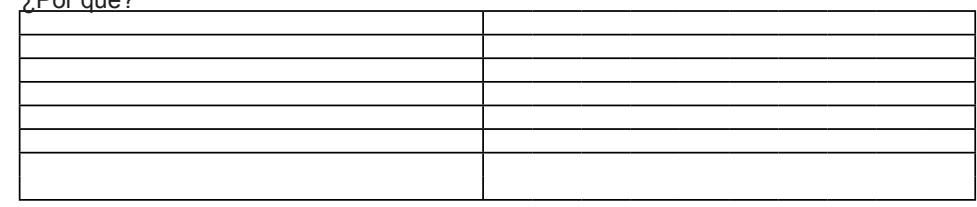

4 ¿Le es difícil encontrar medicamentos genéricos en farmacias y boticas?

Sí

A veces

No

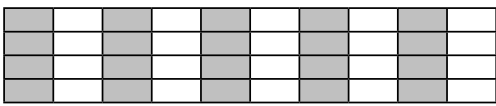

$5 \mathrm{Si}$ el médico le receta un medicamento de marca y en la farmacia le ofrecen cambiárselo por un genérico, ¿aceptaría el cambio?

o Definitivamente no

Probablemente no

Quizás

Probablemente sí

Definitivamente sí

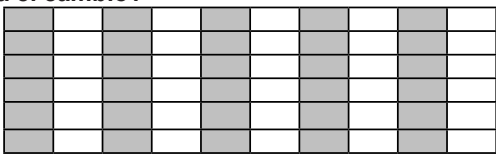

6 ¿Le preocupa que no sea un medicamento seguro y confiable?

Definitivamente no

Probablemente no

A veces

Probablemente sí

Definitivamente sí

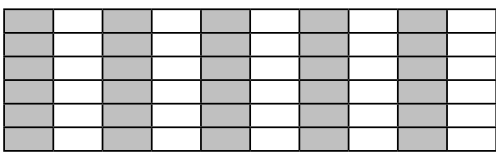

7 ¿Teme que no pueda proporcionarle los beneficios esperados?

Definitivamente no

Probablemente no

A veces

Probablemente sí

Definitivamente sí

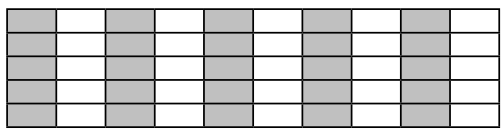

8 ¿Se queda intranquilo al comprar medicamentos genéricos?

Definitivamente no

Probablemente no

A veces

Probablemente sí

Definitivamente sí

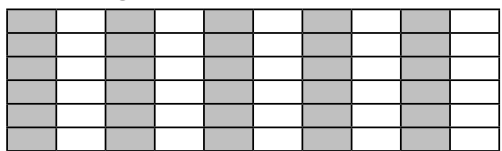

9 ¿El estado le ha facilitado información de los medicamentos genéricos? Sí

No

10 ¿De quien preferiría recibir información sobre medicamentos?

Médico

Químico Farmacéutico

Estado

Laboratorios Farmacéuticos

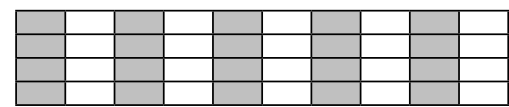

11 Que medio le parecería conveniente para informarse sobre medicamentos

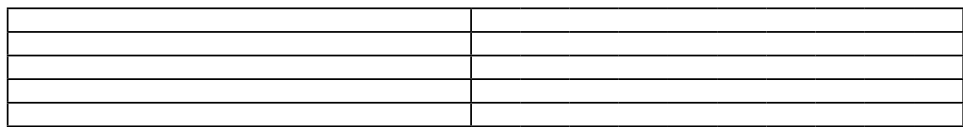

\section{REFERENCIAS BIBLIOGRÁFICAS}

1. Ugarte Oscar: El 10\% de las medicinas que se vende es genérico. [En línea] Acceso 11 Febrero 2011. Disponible en: http://www.adifan.org. pe/web/principal.php/prensa/ngz2.

2. Tobar F. Economía de los medicamentos genéricos en América Latina. Rev Panam Salud Pública 2008; 23(1):59-67.

3. Sheppard Allan. Los medicamentos genéricos: Elementos clave en la sostenibilidad de los sistemas sanitarios a largo plazo. IMS health. E.E.U.U., 2010. p. 9-35.

4. Asociación Peruana de Investigación de Mercado (APEIM). Distribución niveles socioeconómicos de Lima Metropolitana. Enero, 2010.

5. Instituto Nacional de Estadística e Informática (INEI). Censos Nacionales 2007: XI de Población y VI de Vivienda. Lima, 2007.

6. Instituto Nacional de Estadística e Informática (INEI). Planos estratificados de Lima metropolitana a nivel de manzana. Lima, 2009.

7. Puig-Junoy, Jaime. Impacto de la regulación de precio de los Medicamentos sobre la competencia en el mercado de genéricos: Valoración de los efectos y necesidad de reforma. Centre de Recerca en Economía i Salut (CRES) Universitat Pompeu Fabra Barcelona. Realizado a iniciativa de la Autoritat Catalana de la Competència (ACCO). España, 2009. p. 15.

8. Aylas Flores C. Evaluación de los precios ofertados de medicamentos esenciales de farmacias y boticas ubicadas en cuatro distritos del cono norte de Lima. [Tesis para optar el titulo profesional de Químico Farmacéutico]. Facultad de Farmacia y Bioquímica, Universidad Nacional Mayor de San Marcos. Lima, 2008. p. 66.

Manuscrito recibido el: 22/06/2012

Aceptado para su publicación el: 10/12/2012

\section{Correspondencia:}

Nombre: Jesús Rumiche Briceño

Dirección: Jr. Puno 1002- Cercado de Lima, Perú e-mail: jrumicheb@unmsm.edu.pe 